\title{
Danshensu, a major water-soluble component of Salvia miltiorrhiza, enhances the radioresponse for Lewis Lung Carcinoma xenografts in mice
}

\author{
HONG-YING CAO ${ }^{*}$, RUI-LIN DING ${ }^{2 *}$, MENG LI $^{2 *}$, MAO-NAN YANG $^{2}$, LING-LIN YANG $^{2}$, \\ JING-BO WU ${ }^{2}$, BO YANG ${ }^{2}$, JING WANG $^{2}$, CUI-LIAN LUO ${ }^{2}$ and QING-LIAN WEN ${ }^{2}$ \\ Departments of ${ }^{1}$ Emergency and ${ }^{2}$ Oncology, \\ The Affiliated Hospital of Luzhou Medical College, Luzhou, Sichuan 646000, P.R. China
}

Received August 3, 2015; Accepted September 15, 2016

DOI: $10.3892 / \mathrm{ol} .2016 .5508$

\begin{abstract}
The molecule 3-(3,4-dihydroxyphenyl)-2-hydroxypropanoic acid (danshensu), a herbal preparation used in traditional Chinese medicine, has been found to possess potential antitumor and anti-angiogenesis effects. The aim of the present study was to investigate the efficacy of the combination of radiation therapy (RT) with danshensu in the treatment of Lewis lung carcinoma (LLC) xenografts, whilst exploring and evaluating the mechanism involved. In total, 8-week old female C57BL/6J mice were randomly assigned into 3 groups to receive: RT, RT + cisplatin and RT + danshensu, respectively, when LLC reached $100-150 \mathrm{~mm}^{3}$. Each group was divided into 7 subgroups according to the different irradiation doses that were administered. Tumor growth curves were created and the sensitization enhancement ratios of the drugs were calculated. The experiment was then repeated, and the 4 groups of tumor-bearing mice were treated with natural saline, danshensu, $\mathrm{RT}+$ danshensu and $\mathrm{RT}$, respectively. The mice were sacrificed on day 7 , and tumor tissue and blood were collected to determine microvessel
\end{abstract}

Correspondence to: Dr Qing-Lian Wen, Department of Oncology, The Affiliated Hospital of Luzhou Medical College, 25 Taiping Street, Luzhou, Sichuan 646000, P.R. China

E-mail:wq173115@163.com

*Contributed equally

Abbreviations: CAIX, carbonic anhydrase IX; CD31, platelet/endothelial cell adhesion molecule; CVD, cardiovascular disease; DDP, cisplatin; HIF-1 $\alpha$, hypoxia-inducible factor-1 $\alpha$; IHC, immunohistochemistry; LLC, Lewis lung carcinoma; MVD, microvessel density; $\mathrm{PGI}_{2}$, prostaglandin $\mathrm{I}_{2}$; RT, radiation therapy; SER, sensitization enhancement ratio; TGD, tumor growth delay; TXA $_{2}$, thromboxane $\mathrm{A}_{2}$; TXB $\mathrm{TX}_{2}$, thromboxane $\mathrm{B}_{2}$; VEGF, vascular endothelial growth factor

Key words: danshensu, radiotherapy, lung cancer, radiosensitizer, radio-resistance density, the expression of proangiogenic factors, and the levels of blood thromboxane $\mathrm{B}_{2}$ and 6-keto-prostaglandin-F1 $\alpha$. Tumor hypoxia was also detected using in vivo fluorescence imaging. With respect to LLC xenografts, treatment with danshensu + RT significantly enhanced the effects of tumor growth inhibition $(\mathrm{P}<0.05)$. Furthermore, tumor vasculature was remodeled and microcirculation was improved, which significantly reduced tumor hypoxia $(\mathrm{P}<0.05)$. The present study demonstrated that danshensu significantly enhanced the radioresponse of LLC xenografts in mice. The mechanism involved may be associated with the alleviation of tumor cell hypoxia following treatment with danshensu + RT, caused by the improvement of tumor microcirculation and the remodeling of tumor vasculature.

\section{Introduction}

Lung cancer is one of the principal causes of cancer-associated mortality in the world. There are expected to be $\sim 1.6$ million new patients with lung cancer and $\sim 1.4$ million lung cancer-associated mortalities per year (1). Radiation therapy (RT) alone, or combined with other therapy performs an important role in the treatment of lung cancer. It has been reported that $\sim 64 \%$ of patients with lung cancer are treated with radiotherapy $(2,3)$.

As opposed to normal vessels, tumor vasculature is pathological, being structurally and functionally anomalous, with faults in the endothelium, pericytic coverage, and basement membrane $(4,5)$. These abnormalities impair the ability of the vessels to deliver oxygen, thereby interfering with the effect of RT (4) and eventually causing lung cancer to become resistant to radiation (5).

Although it has long been assumed that angiogenic inhibitors destroy blood vessels and lead to hypoxia, thus reducing the effect of RT, a substantial amount of experimental and clinical evidence has demonstrated that the combined use of RT and angiogenesis inhibitors could be beneficial in terms of overcoming the resistance of angiogenesis inhibitors and improving tumor response to RT $(6,7)$. A rational basis for the mechanism involved is that angiogenesis inhibitors normalize the tumor vasculature, which has been defined as 
selective pruning of excess tumor vessels via the inhibition of endothelial cell proliferation, migration or invasion, tube formation, and by initiating endothelial cell apoptosis (6). The best radioresponse was achieved during this normalization window (7). However anti-angiogenic therapy is not an effective therapy due to tumor functional resistance (8). Therefore, the development of novel therapeutic strategies is essential.

The molecule 3-(3,4-dihydroxy-phenyl)-2-hydroxy-p ropinic acid (danshensu) is the first water-soluble phenolic compound found and isolated from Salvia miltiorrhiza that has been demonstrated to be effective in terms of activating blood circulation and reducing blood stasis (9). It has been demonstrated that danshensu may possess the potential to enhance microcirculation, suppress the formation of reactive oxygen species, constrain platelet adhesion and aggregation and impart cardiovascular protection $(10,11)$. Notably, certain studies provide evidence that danshensu could attenuate the increased endothelial permeability of tumor vasculature, and suppress the expression of vascular endothelial growth factor (VEGF) $(12,13)$. It is therefore reasonable to suppose that danshensu may exert an effect on aberrant tumor vasculature and microcirculation, working as a radiosensitizer.

In order to confirm the hypothesis of the present study, Lewis lung carcinoma (LLC) mice models were used to observe tumor growth under different treatments, including danshensu and RT. The tumor tissue and blood samples harvested from tumor-bearing mice were collected to investigate this mechanism.

\section{Materials and methods}

Cell lines, cell culture and reagents. LLC cells were obtained from The American Type Culture Collection (Manassas, VA, USA) and maintained in Dulbecco's modified Eagle medium (Thermo Fisher Scientific, Inc., Waltham, MA, USA) with $10 \%$ heat-inactivated fetal bovine serum (Thermo Fisher Scientific, Inc.), $100 \mathrm{U} / \mathrm{ml}$ penicillin and $100 \mathrm{U} / \mathrm{ml}$ streptomycin (Tianjin Zhongan Pharmaceutical Co., Ltd., Tianjin, China), and kept in a humidified $5 \% \mathrm{CO}_{2}$ atmosphere incubator at $37^{\circ} \mathrm{C}$.

Animals and tumor models. The female C57BL/6J mice were 8 weeks old with weights of $19.16 \pm 1.95 \mathrm{~g}$, and were provided by the Laboratory Animal Center of Chongqing municipality (Chongqing, China). The mice were administered sterile food pellets and water ad libitum, and were kept in specific pathogen free laminar airflow boxes. All the animal care and experimental procedures followed were approved by the Institutional Animal Care and Use Committee of Luzhou Medical College (no., SYXK2013-181; Luzhou, China). The xenografts were established subcutaneously via the injection of LLC cells $\left(0.2 \mathrm{ml} ; 1 \times 10^{6}\right.$ cells) into the right thighs of the mice.

Treatment protocol. When the tumor diameter, measured by calipers, reached $100-150 \mathrm{~mm}^{3}$, a total of 168 mice were divided into 3 treatment groups: RT alone; cisplatin (DDP) + RT; and danshensu + RT. Each group consisted of 7 subgroups that received one dose of total irradiation of $0,2,4,6,8$, 16 and $24 \mathrm{~Gy}$, respectively. The drugs were administered by intraperitoneal (IP) injection at a dose of $60 \mathrm{mg} / \mathrm{kg}$ for the 2 combination groups. In the RT alone group, the same volume of phosphate-buffered saline was injected. The feasibility of the injection dose was examined in the preliminary experiments. Briefly, doses of 20, 40, 60 and $80 \mathrm{mg} / \mathrm{kg}$ were selected for the preliminary experiments according to certain studies $(14,15)$. At $60 \mathrm{mg} / \mathrm{kg} / \mathrm{day}$, danshensu exerted the maximum antitumor effect, therefore, this dose was selected for use in the combination study with irradiation, which was diluted in $0.2 \mathrm{ml}$ sterile $0.9 \%$ normal saline. Irradiation was performed for 30 min subsequent to DDP or danshensu injection. Prior to RT, the mice were anesthetized with $1 \%$ pentobarbital at $40 \mathrm{mg} / \mathrm{kg}$ via IP injection and restrained on an acrylic board via adhesive tape. Using 2 lead pallets to shield the bodies of the mice, only the tumor-bearing positions were irradiated locally in the middle of the $24 \mathrm{~cm}$ field. RT was performed using the GWXJ80 ${ }^{60} \mathrm{Co}$ Teletherapy Unit at a dose rate of $36.45 \mathrm{cGy} / \mathrm{min}$ (Oncology Department, the Affiliated Hospital of Luzhou Medical College, Luzhou, China). A $2.0 \mathrm{~cm}$ bolus of tissue equivalent material was used to achieve a homogeneous dose deposition in the tumor.

The experiment was then repeated, and the irradiation dose of $10 \mathrm{~Gy}$ was selected based on the previous results of the aforementioned experiment. A total of 24 mice were involved in the second study. The tumor models were established as mentioned above. When the size of the tumor reached $100-150 \mathrm{~mm}^{3}$, the mice were randomly divided into 4 groups, with 6 mice in each group. The 4 groups of tumor-bearing mice were treated with an IP injection of natural saline $(0.2 \mathrm{ml}$ each), danshensu at $60 \mathrm{mg} / \mathrm{kg}$, RT (10 Gy each), or danshensu + RT, respectively. The drugs were administered for 7 consecutive days and irradiation was performed 30 min subsequent to the last administration. The mice from each group were sacrificed by cervical dislocation on day 7. Using the tumor tissues collected from each group, additional analyses were performed, focusing on changes of microvessel density (MVD) and tumor hypoxia. Mice blood samples were collected to additionally detect thromboxane (TX) $\mathrm{B}_{2}$ and 6-keto-prostaglandin (PG) F1a by ELISA.

Tumor response evaluation. Tumor response was assessed using a tumor-growth delay (TGD) assay. TGD, in days, is defined as the difference between $T_{5 v 0}$ of treated tumors compared with that of the untreated tumors. $T_{5 \mathrm{v} 0}$ represented the number of days required for the tumor to grow from its original size to 5-times its original volume. The group of mice treated with 0 Gy alone was regarded as the untreated group. The tumor volumes were calculated as: $\mathrm{V}=\mathrm{a} \mathrm{x} \mathrm{b}^{2} \mathrm{x} \pi / 6$ where (a) is the larger and (b) is the perpendicular shorter tumor axis, measured 3 times a week until day 30. A tumor growth curve was created based on tumor size and number of survival days following irradiation.

To detect whether danshensu could enhance the radioresponse of LLC models, the concept of sensitization enhancement ratio (SER) was introduced in the present study. The SER represented the ratio of irradiation dose required to reach the endpoint in the irradiation-only group vs. the irradiation dose required in the combination groups. A result $>1$ indicated that the drug enhanced the radioresponse. Additionally, as an indirect measurement of the general toxicity of each treatment regimen, the weights of the mice were recorded. 
Immunohistochemistry (IHC) analysis. The tumor tissues were fixed in $10 \%$ formalin at room temperature for $24 \mathrm{~h}$, embedded in paraffin and sectioned at $5 \mu \mathrm{m}$ thickness. Following microwave treatment in citrate buffer $(\mathrm{pH} 6.0)$, the slides were incubated in $3 \%$ hydrogen peroxide for $20 \mathrm{~min}$ to prevent endogenous peroxidase activity. Subsequent to 3 rinses in phosphate-buffered saline, the sections were incubated with rabbit anti-mouse monoclonal antibodies for platelet/endothelial cell adhesion molecule (CD31; dilution, 1:100; catalogue no., bs-20322R; Beijing Biosynthesis Biotechnology Co. Ltd., Beijing, China), vascular endothelial growth factor (VEGF; dilution, 1:50; catalogue no., bs-1957R; Beijing Biosynthesis Biotechnology Co.Ltd.), and hypoxia-inducible factor (HIF)-1 $\alpha$ (dilution, 1:50; catalogue no., bs-20398R; Beijing Biosynthesis Biotechnology Co. Ltd.), and then incubated with horseradish peroxidase (HRP)-conjugated secondary antibodies (dilution, 1:2,000; catalogue no., bs-0295G-HRP; Beijing Biosynthesis Biotechnology Co. Ltd.). Next, the slides were developed using a diaminobenzidine HRP Color Development Kit (Beyotime Institute of Biotechnology, Haimen, China) and counterstained with hematoxylin. The images were analyzed by Image-Pro Plus 6.0 (Media Cybernetics, Inc., Rockville, MD, USA).

ELISA. Plasma $\mathrm{TXB}_{2}$ and 6-keto-PGF1 $\alpha$ levels were detected by an ELISA kit according to the manufacturer's protocol (Beijing Biosynthesis Biotechnology Co. Ltd.). Calibration curves were made using known concentrations of vascular endothelial contraction factors. The colorimetric reaction was read with a Benchmark Microplate Reader (Benchmark Electronics, Angleton, TX, USA).

In vivo fluorescence imaging. An IVIS Imaging System (PerkinElmer, Inc., Waltham, MA, USA) was used to investigate variations in tumor cell hypoxia. In total, 20 tumor-bearing mice were divided into 4 groups: Control; danshensu at $60 \mathrm{mg} / \mathrm{kg}$; RT (10 Gy each); and danshensu + RT. Prior to imaging, the mice were injected with HypoxiSense 680 (100 $\mu \mathrm{l}$; catalogue no., NEV11070; PerkinElmer, Inc.) in the tail vein. HypoxiSense 680 is a fluorescent reagent that targets the carbonic anhydrase IX (CAIX) of tumor cells in hypoxia-affected areas (16). Subsequently, $24 \mathrm{~h}$ post-injection, the mice were anesthetized with pentobarbital and the hair on the tumors was removed. The mice were then restrained on an imaging plate. Imaging was performed and the fluorescence signal was calculated using IVIS Imaging Software (PerkinElmer, Inc.).

Data analysis. The continuous variables were expressed as the mean \pm standard deviation, and significance between groups was calculated using a one-way analysis of variance. The average number of pairwise comparisons was calculated using Tamhane's $\mathrm{T}_{2}$ test. $\mathrm{P}<0.05$ was considered to indicate a statistically significant difference. Data analyses were performed using the IBM SPSS (Armonk, NY, USA) statistics 19.0 software.

\section{Results}

Danshensu enhanced radioresponse of LLC mice models. The tumor growth curves are exhibited in Fig. 1 and TGD is demonstrated in Table I. Tumor growth slowed as irradiation dose increased, and TGD was gradually extended in the RT alone, danshensu + RT, and DDP + RT groups. This phenomenon became evident when the irradiation dose reached $8 \mathrm{~Gy}$.

Dose-response curves were generated for the RT alone, danshensu + RT, and DDP + RT groups from the data in Table I. As shown in Fig. 2, the drug treatments combined with RT prolonged TGD, with a statistically significant difference in the TGD compared with RT alone $(\mathrm{P}=0.003)$. In addition, the 3 dose-response curves were almost parallel between 10 and 20 days, indicating a similar variation trend among the 3 curves during this period. Thus, a TGD of 15 days was taken as the biological endpoint. To achieve a TGD of 15 days, 18.614, 10.651 and $7.335 \mathrm{~Gy}$ were required for the RT alone, danshensu + RT, and DDP + RT groups, respectively. Therefore, the SER was $1.748(18.614 / 10.651)$ for danshensu and 2.538 (18.614/7.335) for DDP. The results suggest that danshensu and DDP enhances the radioresponse of LLC mice models, however, the effect of danshensu was weaker. It is of note that the mice tolerated all the therapies, with only minor weight fluctuations during the treatment period (data not shown).

Danshensu $+R T$ could improve tumor cell hypoxia. The present study sought to determine if danshensu + RT could reduce tumor cell hypoxia. The in vivo imaging was performed using a CAIX-targeted fluorescent reagent. Fig. 3A shows the fluorescent reagent labeled on the hypoxia regions in mice models. It is clear to see that the fluorescent reagent was stably expressed in the hypoxia region and was degraded in the normal oxygen regions. Levels of hypoxia were severe in the control, RT alone and danshensu alone groups, with the photon counts $643.9 \pm 162.8,579.6 \pm 49.3$ and $469.97 \pm 33.05$, respectively. Although the photon counts in the RT alone and danshensu alone groups were lower compared with those in the control group, the difference did not prove to be statistically significant $(P>0.05)$. However, the photon count of the danshensu + RT group was 252.6 \pm 51.3 , indicating that the combination of danshensu + RT could significantly alleviate tumor cell hypoxia, compared with the control $(\mathrm{P}=0.001)$, RT alone $(\mathrm{P}=0.002)$ and danshensu alone groups $(\mathrm{P}=0.019)$, respectively (Fig. 3B).

To additionally validate the findings of the present study, the expression of HIF-1 $\alpha$ in LLC xenografts was detected using IHC staining (Fig. 4A). As shown in Fig. 4B, the expression level of HIF-1 $\alpha$ in the danshensu + RT group was significantly lower compared with in the 3 other groups $(\mathrm{P}<0.001)$. However, the expression level of HIF-1 $\alpha$ in the tumor tissue of the danshensu alone group was similar to that of the control group $(\mathrm{P}=0.101)$.

Danshensu + RT could remodel tumor vasculature. An IHC examination of various tumor sections was performed to determine MVD and the expression levels of VEGF. The tumor sections were stained with anti-CD31 antibody, a vascular endothelial marker. Compared to the control, a significant reduction of MVD was observed in the RT, danshensu and RT + danshensu-treated groups $(\mathrm{P}<0.001)$. In addition, a statistical significance was found between the danshensu + RT group and the RT alone group in terms of microvessel density. The combination group had an even greater effect in terms of decreasing 
Table I. Tumor growth delay in Lewis Lung Cancer xenografts under different radiation modalities and radiation dose.

\begin{tabular}{lccccr}
\hline & \multicolumn{3}{c}{$\mathrm{T}_{5 \mathrm{v} 0}$ (days) } & \multicolumn{2}{c}{ TGD (days) } \\
\cline { 2 - 4 } $\begin{array}{l}\text { Radiation } \\
\text { dose, Gy }\end{array}$ & $\begin{array}{c}\text { RT } \\
\text { alone }\end{array}$ & $\begin{array}{c}\text { Danshensu } \\
\text { plus RT }\end{array}$ & $\begin{array}{c}\text { DDP } \\
\text { plus RT }\end{array}$ & $\begin{array}{c}\text { RT } \\
\text { alone }\end{array}$ & $\begin{array}{c}\text { Danshensu } \\
\text { plus RT }\end{array}$ \\
\hline 0 & 4.75 & 4.95 & 6.05 & 0.00 & 0.20 \\
2 & 6.45 & 6.05 & 9.05 & 1.70 & 1.30 \\
plus RT \\
4
\end{tabular}

DDP, Cisplatin; RT, radiotherapy; $\mathrm{T}_{5 \mathrm{v}}$, the days required for tumor growth from the original size to 5 times its original volume; TGD, tumor growth delay.


Figure 1. Tumor growth curves treated with a single dose of irradiation, danshensu + RT, or DDP + RT. (A) Tumor treated with a single dose of irradiation. (B) Tumor treated with $60 \mathrm{mg} / \mathrm{kg}$ of danshensu followed by irradiation. (C) Tumor treated with $60 \mathrm{mg} / \mathrm{kg}$ of DDP followed by irradiation. As irradiation dose increased, tumor growth in each group became slow, as became apparent when the irradiation dose reached 8 Gy. DDP, cisplatin; RT, radiation therapy.

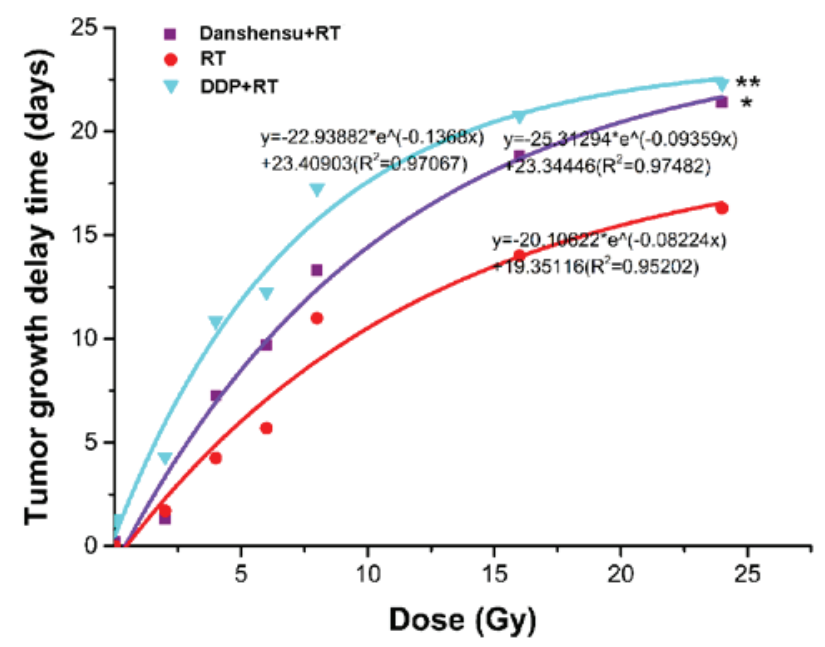

Figure 2. Dose-response curves for RT alone, danshensu + RT, or DDP + RT in Lewis lung carcinoma. Danshensu and DDP combined with RT prolonged tumor growth delay, with a statistically significant difference compared with RT alone. ${ }^{*} \mathrm{P}=0.003$ vs. $\mathrm{RT} ;{ }^{* *} \mathrm{P}<0.001$ vs. RT. DDP, cisplatin; RT, radiation therapy.

MVD ( $\mathrm{P}<0.001$, Fig. 5D). Similar results were found with respect to the expression of VEGF. Compared to the control, the level of VEGF significantly reduced following treatment with danshensu alone $(\mathrm{P}<0.001)$, and additionally reduced following treatment with danshensu + RT $(\mathrm{P}<0.001$, Fig. $5 \mathrm{~A}$ and $\mathrm{B}$ ). The results of the present study demonstrate that the alleviation of tumor cell hypoxia caused by danshensu + RT was associated with their effect on tumor vasculature.

Danshensu + RT could improve microcirculation of solid tumors. An ELISA assay was used to detect the serum concentrations of $\mathrm{TXB}_{2}$ and 6-keto-PGF1 $\alpha$ (Fig. 6). In the LLC xenografts of the danshensu + RT, RT alone and danshensu alone groups there was no significant change in the concentrations of $\mathrm{TXB}_{2}$ or 6-keto-PGF1 $\alpha$ compared with the control group following treatment $(\mathrm{P}>0.05)$. However, for serum $\mathrm{TXB}_{2}$ concentration, there was a statistically significant difference between the RT alone and the danshensu + RT groups 
A

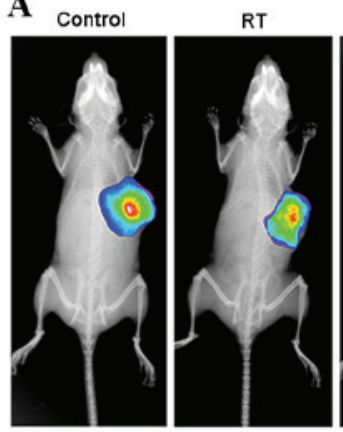

Danshensu

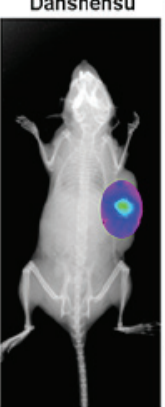

Danshensu+RT

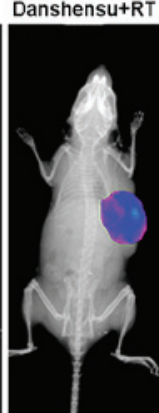

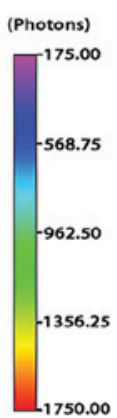

B

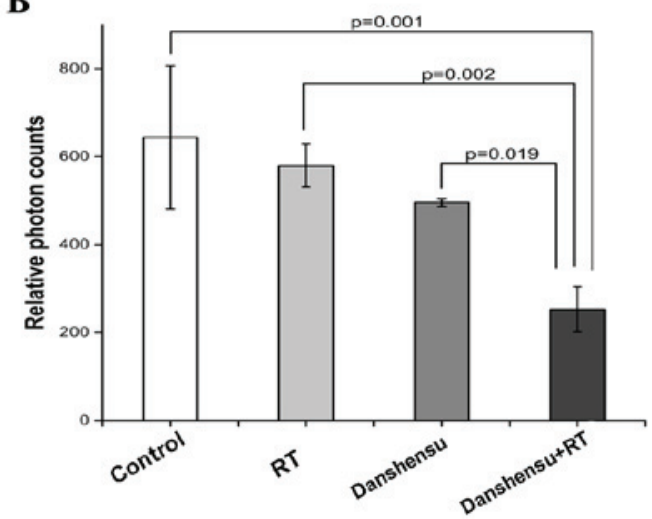

Figure 3. Effect of different treatment modalities on CAIX expression-associated fluorescence. (A) Representative subcutaneous tumor model imaging of light intensity of HypoxiSense 680 distribution subsequent to tail-vein injection. The groups were control, danshensu at $60 \mathrm{mg} / \mathrm{kg}, \mathrm{RT}(10 \mathrm{~Gy}$ each) and danshensu + RT. The fluorescent reagent was stably expressed in the hypoxia regions and was degraded in the normal oxygen regions. All the images were represented under the same color scale. (B) The photon counts of the tumor area in treated mice. The photon counts of danshensu + RT were much lower compared with the other 3 groups. Data are expressed as the mean \pm standard deviation. CAIX, carbonic anhydrase IX; RT, radiation therapy.
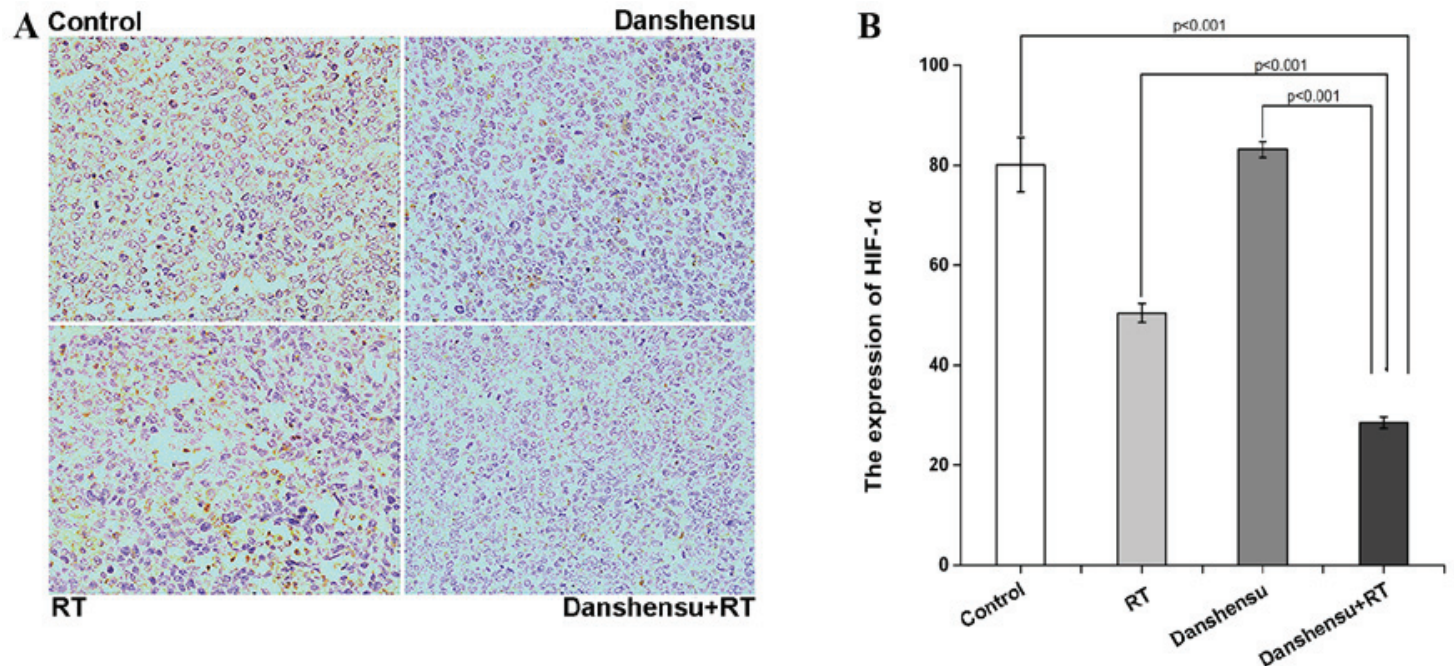

Figure 4. The expression of HIF-1 $\alpha$ in Lewis lung carcinoma xenograft tissue harvested from mice sacrificed on day 7 subsequent to the treatment initiation. The mice were treated with either $0.2 \mathrm{mml}$ natural saline, danshensu at $60 \mathrm{mg} / \mathrm{kg}, 10 \mathrm{~Gy}$ RT or danshensu + RT. (A) Representative images stained by an antibody to HIF-1 $\alpha$ in tumor tissues harvested from 6 mice in each group (magnification, $\mathrm{x} 400$ ). (B) Histogram of expression of HIF-1 $\alpha$ in tumor tissues. The expression level of HIF-1 $\alpha$ in the danshensu + RT group was significantly reduced compared to the 3 other groups (P<0.001). The mean optical density of the immunohistochemical images was determined using Image-Pro Plus software. Data is expressed as the mean \pm standard deviation. HIF-1 $\alpha$, hypoxia-inducible factor-1 $\alpha$; RT, radiation therapy.

$(\mathrm{P}<0.001)$. Furthermore, the ratio of $\mathrm{TXB}_{2}$ to 6-keto-PGF1 $\alpha$ ( $\mathrm{T} / \mathrm{P}$ ratio) was 0.517 for danshensu $+\mathrm{RT}$ group and 1.289 for RT alone group. The results of the present study indicate that the microcirculation of solid tumors improved following a combined treatment modality of RT with danshensu.

\section{Discussion}

As a type of natural medicine, Salviae Miltiorrhizae has been widely used for a long time. The present study focused on the potential radiosensitizing effect of danshensu, the main water-soluble component of the aforementioned medicine, and found that danshensu significantly enhanced the radioresponse of LLC xenografts in mice.

The present study supports the hypothesis that danshensu + RT significantly increases the effects of tumor growth inhibition in a mouse model under different irradiation doses. Notably, compared to the RT alone group, the danshensu + RT group significantly prolonged TGD. Additionally, the SER of danshensu was 1.748, while that of DDP was 2.538. The results of the present study support previous studies that demonstrated that DDP could enhance radioresponse $(17,18)$. Furthermore, the present study found that danshensu also exhibits a radiosensitizing effect on LLC. No immediate or long-term unspecific toxicity during the treatment or follow-up periods were observed in the present study.

The microenvironment of solid tumors is different from that of normal tissues, and is characterized by hypoxia, acidosis and excessive vessel leakiness (4). Hypoxia is known to be the most important characteristic in the tumor microenvironment. Increasing evidence supports the theory that hypoxia is associated with radioresistance, tumor recurrence, 

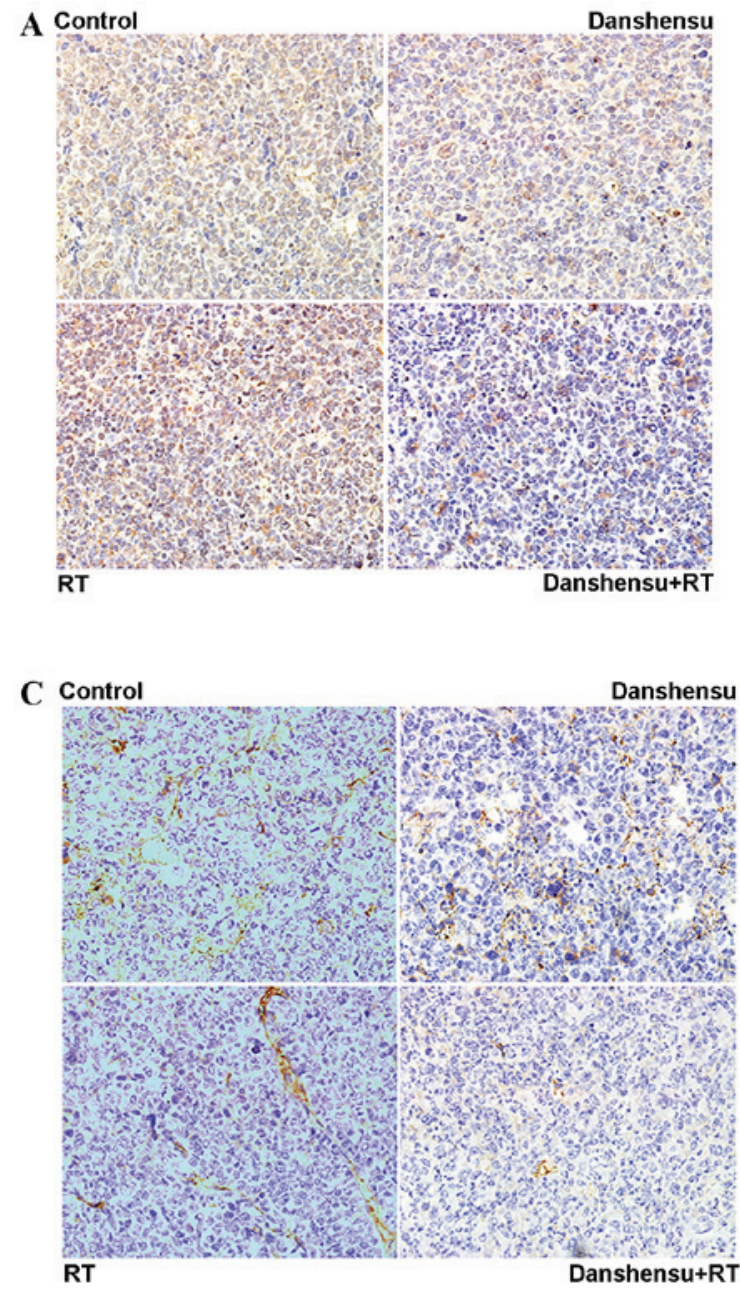

B

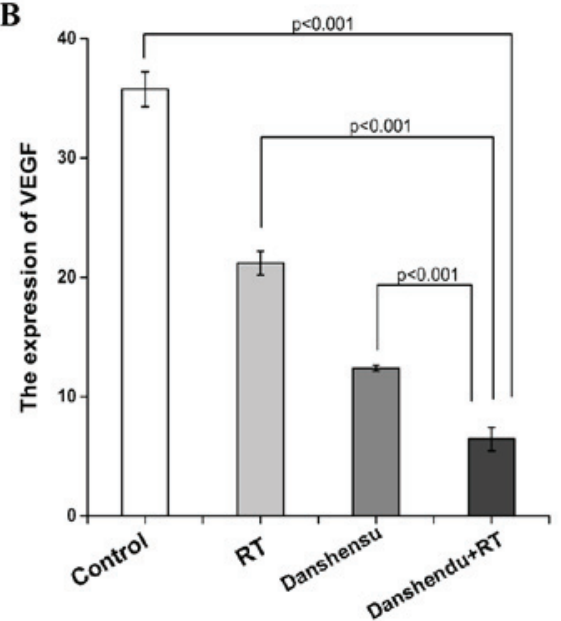

D

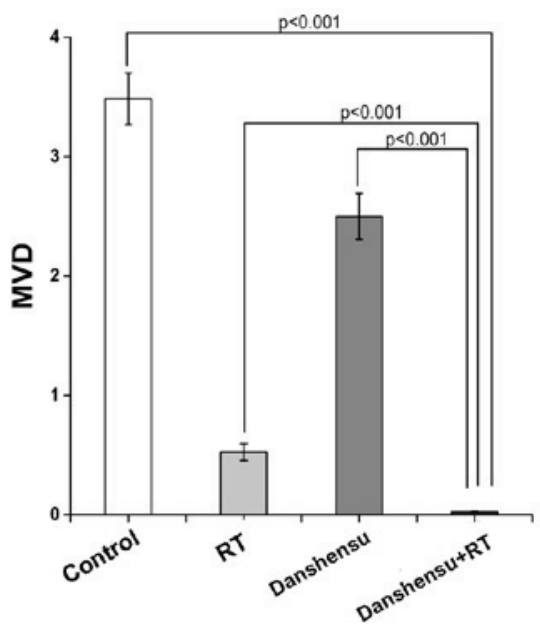

Figure 5. The analyses of MVD and expression of VEGF by IHC. The mice were either treated with $0.2 \mathrm{ml}$ natural saline, danshensu at $60 \mathrm{mg} / \mathrm{kg}, 10 \mathrm{~Gy}$ RT or danshensu + RT. (A) Representative images stained by an antibody to VEGF in tumor tissues harvested from mice sacrificed on day 7 subsequent to initial treatment in each group (magnification, x400). (B) Histogram of expression of VEGF in tumor tissues. The level of expression of VEGF in the danshensu + RT group was significantly reduced compared to the 3 other groups $(\mathrm{P}<0.001)$. (C) Representative images stained by an antibody to CD31 in tumor tissues harvested from mice sacrificed on day 7 subsequent to initial treatment in each group (magnification, $x 400$ ). (D) Histogram of the expression of MVD-CD31 in tumor tissues. A significant reduction of MVD was observed in the danshensu + RT group compared to the 3 other groups $(\mathrm{P}<0.001)$. The mean optical density of the IHC image was determined using Image-Pro Plus software. Data is expressed as the mean \pm SD. CD31, platelet/endothelial cell adhesion molecule; IHC, immunohistochemistry; MVD, microvessel density; RT, radiation therapy; VEGF, vascular endothelial growth factor.

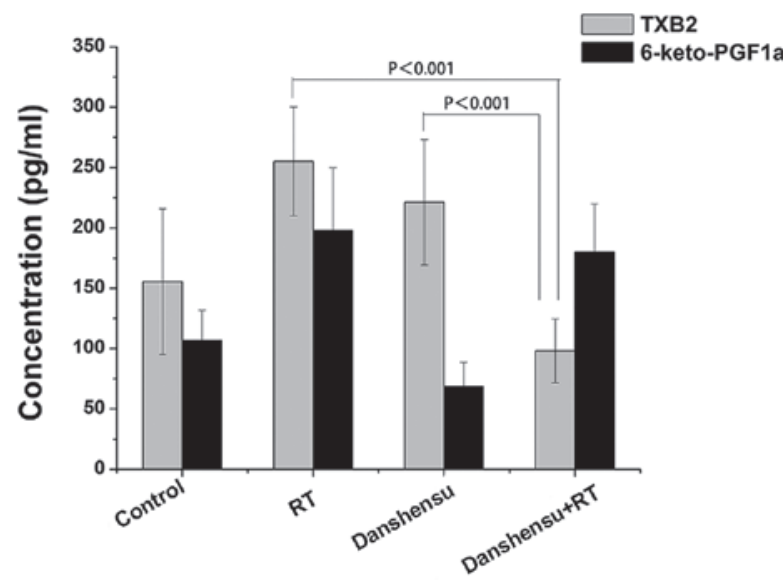

Figure 6. Concentrations of blood plasma $\mathrm{TXB}_{2}$ and 6-keto-PGF1 $\alpha$ of each group. Compared with the RT alone group, a significant reduction of the level of $\mathrm{TXB}_{2}$ was observed in the danshensu + RT group. Data is expressed as the mean \pm standard deviation. $\mathrm{PGI}_{2}$, prostaglandin $\mathrm{I}_{2} ; \mathrm{RT}$, radiation therapy; $\mathrm{TXB}_{2}$, thromboxane $\mathrm{B}_{2}$. and poor prognosis $(19,20)$. Therefore, in order to investigate the underlying mechanism of the enhanced radiosensitivity caused by danshensu, additional research is required on these factors.

Tumor vasculature is aberrant compared with that in normal tissue, as it is hyperpermeable, tortuous and with high interstitial pressure. It has been reported that the abnormal tumor vessels are mainly mediated by VEGF signaling (21). The agents targeted by VEGF and its receptor, or other angiogenic factors, could normalize abnormal tumor vessels and alleviate tumor cell hypoxia, enhancing the radioresponse of tumor growth in solid tumors $(22,23)$.

Danshensu has been reported to be an effective therapy for cardiovascular disease (CVD) (24). The mechanism of how danshensu ameliorates microcirculation in CVD has been described in numerous studies. It can promote blood circulation, reduce blood stasis, improve blood flow in coronary arteries, relax vascular muscle and scavenge thrombi (25-28). Notably, certain studies provide evidence that danshensu 
exhibits potential anti-tumor and anti-angiogenesis effects. Zhang et al (13) found that danshensu had anti-tumor activity by affecting tumor angiogenesis and tumor invasion. Zhang's study pointed out that danshensu demonstrated great efficacy in inhibiting the expression of VEGF in a tumor cell line in vitro. Tian et al (29) also found that danshensu could reduce the expression of VEGF in the process of vascular proliferative retinopathy of rabbits, inhibiting the formation of new vessels. The present study observed that danshensu could also downregulate the expression of VEGF and decrease MVD levels in LLC mice models, indicating significant danshensu-induced inhibition of tumor vessels. The results of the present study confirmed that danshensu could work as an angiogenic inhibitor and that its radiosensitizing effect may be associated with its anti-angiogenic activity.

The alleviation of tumor cell hypoxia following treatment with danshensu alone was not observed in the present study. However, danshensu + RT exhibited a significant effect on hypoxia. The 2 methods used to investigate hypoxia in the LLC mouse model in the present study were: Detecting the expression of HIF-1 $\alpha$ by IHC; and detecting CAIX expression-associated fluorescence using the IVIS imaging system. HIF-1 $\alpha$ is the key factor that mediates hypoxic responses. Furthermore, CAIX is induced by hypoxia and performs a role in tumor growth (16). The overexpression of CAIX has been reported in a wide variety of human carcinomas including lung cancer (30). The results of the present study were not consistent with the expectation of the present authors that danshensu could alleviate tumor cell hypoxia such as other angiogenic agents. The reason may be associated with drug dose or frequency of drug administration, so additional study is required to clarify this point. However, the combined use of danshensu and irradiation improved tumor cell hypoxia.

The underlying mechanism of the hypoxic change caused by danshensu + RT was investigated. MVD and the expression of VEGF significantly decreased subsequent to treatment with danshensu + RT. As aforementioned, danshensu exhibits a potent anti-angiogenic effect. Irradiation could also damage the endothelial cells, reducing MVD levels (31). However, danshensu + RT appeared to exhibit a stronger effect on tumor vasculature compared to other groups. VEGF is a key angiogenic factor. The inhibition of VEGF expression may lead to decreased angiogenesis in tumors. In addition, MVD is a useful prognostic predictor, as it represents the degree of tumor vascularization in cancer patients (32). The results of the present study suggest that the combination of $\mathrm{RT}+$ danshensu may produce a synergistic effect in terms of inhibiting tumor vasculature. When combined with RT, danshensu could contribute towards the normalization of the vascular structure. Furthermore, the 2 therapies together may inhibit aberrant tumor vessels, which may also contribute to the improvement of tumor cell hypoxia. Therefore, the alleviation of tumor cell hypoxia caused by danshensu + RT works at least in part via tumor vasculature remodeling.

The present study also found that danshensu + RT may affect the microcirculation of solid tumors, and this mechanism may also be associated with hypoxic change. The serum concentrations of $\mathrm{TXB}_{2}$ and 6-keto-PGF1 $\alpha$ were detected using ELISA. Levels of $\mathrm{TXB}_{2}$ were significantly lower following treatment with RT + danshensu compared with treatment of RT alone in the blood samples. $\mathrm{TXB}_{2}$ and 6-keto-PGF1 $\alpha$ are the stable metabolites of $\mathrm{TXA}_{2}$ and $\mathrm{PGI}_{2}$, released by vascular endothelial cells $(33,34)$. These 2 bio-activators are important in terms of regulating vascular tension. In physiologically stable conditions, the levels of $\mathrm{TXA}_{2}$ and $\mathrm{PGI}_{2}$ maintain a relative balance $(34,35)$, however, when blood vessels are injured by certain factors, including $\mathrm{RT}$, an increased $\mathrm{TXB}_{2}$ level and $\mathrm{TXB}_{2} / 6$-keto-PGF1 $\alpha(\mathrm{T} / \mathrm{P})$ ratio will be observed (36). The present study observed an increase in $\mathrm{TXB}_{2}$ level subsequent to irradiation treatment. Furthermore, the T/P ratio was 0.517 for the danshensu + RT group, and 1.289 for the RT alone group, indicating that danshensu exhibits an effect on the high serum $\mathrm{TXB}_{2}$ level caused by RT. The results of the present study support the hypothesis that danshensu may regulate the imbalance between $\mathrm{TXB}_{2}$ and 6-keto-PGF1 $\alpha$ that is caused by RT. As a result of treatment with danshensu, blood vessels will expand, and microcirculation will be improved, thus hypoxia in tumor areas will be alleviated.

In summary, the present study demonstrated that danshensu + RT significantly prolonged TGD and inhibited tumor growth. Danshensu enhanced the radioresponse of LLC in mice models. The mechanism of this may be associated with the alleviation of tumor cell hypoxia following treatment with danshensu + RT, caused by the improvement of tumor microcirculation and the remodeling of tumor vasculature.

\section{Acknowledgements}

This study was supported by the Science and Technology Board Foundation of Luzhou, China (grant no., 2014-41). The authors thank all colleagues at The Laboratory of Luzhou Medical College (Luzhou, China) who provided their assistance for the present study. The authors also thank the anonymous referee for his/her helpful comments, which notably improved the quality of the present study.

\section{References}

1. Chen W, Zheng R, Zhang S, Zhao P, Zeng H, Zou X and He J: Annual report on status of cancer in China, 2010. Chin J Cancer Res 26: 48-58, 2014.

2. Tyldesley S, Boyd C, Schulze K, Walker H and Mackillop WJ: Estimating the need for radiotherapy for lung cancer: An evidence-based, epidemiologic approach. Int J Radiat Oncol Biol Phys 49: 973-985, 2001.

3. Siegel R, Naishadham D and Jemal A: Cancer statistics, 2012. CA Cancer J Clin 62: 10-29, 2012.

4. Jain RK: Normalizing tumor microenvironment to treat cancer: Bench to bedside to biomarkers. J Clin Oncol 31: 2205-2218, 2013.

5. Vaupel P: Tumor microenvironmental physiology and its implications for radiation oncology. Semin Radiat Oncol 14: 198-206, 2004.

6. Winkler F, Kozin SV, Tong RT, Chae SS, Booth MF, Garkavtsev I, Xu L, Hicklin DJ, Fukumura D, di Tomaso E, et al: Kinetics of vascular normalization by VEGFR2 blockade governs brain tumor response to radiation: Role of oxygenation, angiopoietin-1, and matrix metalloproteinases. Cancer Cell 6: 553-563, 2004

7. Meng MB, Jiang XD, Deng L, Na FF, He JZ, Xue JX, Guo WH, Wen QL, Lan J, Mo XM, et al: Enhanced radioresponse with a novel recombinant human endostatin protein via tumor vasculature remodeling: Experimental and clinical evidence. Radiother Oncol 106: 130-137, 2013.

8. Dey N, De P and Brian LJ: Evading anti-angiogenic therapy: Resistance to anti-angiogenic therapy in solid tumors. Am J Transl Res 7: 1675-1698, 2015. 
9. Chen SJ and Ren JL: Identification of a potential anticancer target of danshensu by inverse docking. Asian Pac J Cancer Prev 15: 111-116, 2014.

10. Yang RX, Huang SY, Yan FF, Lu XT, Xing YF, Liu Y, Liu YF and Zhao YX: Danshensu protects vascular endothelia in a rat model of hyperhomocysteinemia. Acta Pharmacol Sin 31: $1395-1400,2010$

11. Zhang N, Zou H, Jin L, Wang J, Zhong MF, Huang P, Gu BQ, Mao SL, Zhang $\mathrm{C}$ and Chen H: Biphasic effects of sodium danshensu on vessel function in isolated rat aorta. Acta Pharmacol Sin 31: 421-428, 2010.

12. Ding M, Ye TX, Zhao GR, Yuan YJ and Guo ZX: Aqueous extract of Salvia miltiorrhiza attenuates increased endothelial permeability induced by tumor necrosis factor-alpha. Int Immunopharmacol 5: 1641-1651, 2005.

13. Zhang LJ, Chen L, Lu Y, Wu JM, Xu B, Sun ZG, Zheng SZ and Wang AY: Danshensu has anti-tumor activity in B16F10 melanoma by inhibiting angiogenesis and tumor cell invasion. Eur J Pharmacol 643: 195-201, 2010.

14. Hu T, Wei G, Xi M, Yan J, Wu X, Wang Y, Zhu Y, Wang C and Wen A: Synergistic cardioprotective effects of Danshensu and hydroxysafflor yellow A against myocardial ischemia-reperfusion injury are mediated through the $\mathrm{Akt} / \mathrm{Nrf} 2 / \mathrm{HO}-1$ pathway. Int J Mol Med 38: 83-94, 2016

15. Chen YC, Cao WW, Cao Y, Zhang L, Chang BB, Yang WL and Liu XQ: Using neural networks to determine the contribution of danshensu to its multiple cardiovascular activities in acute myocardial infarction rats. J Ethnopharmacol 138: 126-134, 2011.

16. Gu MJ and Kwon KW: Carbonic anhydrase IX expression is associated with favorable prognostic factors in small intestinal carcinoma. J Histochem Cytochem 62: 205-210, 2014.

17. Akudugu JM and Slabbert JP: Modulation of radiosensitivity in Chinese hamster lung fibroblasts by cisplatin. Can J Physiol Pharmacol 86: 257-263, 2008.

18. Zhang X, Yang H, Gu K, Chen J, Rui M and Jiang GL: In vitro and in vivo study of a nanoliposomal cisplatin as a radiosensitizer. Int J Nanomedicine 6: 437-444, 2011.

19. Yoshimura $M$, Itasaka $S$, Harada $H$ and Hiraoka $M$ : Microenvironment and radiation therapy. Biomed Res Int 2013: 685308, 2013.

20. Kizaka-Kondoh S, Tanaka S, Harada H and Hiraoka M: The HIF-1-active microenvironment: An environmental target for cancer therapy. Adv Drug Deliv Rev 61: 623-632, 2009.

21. Peng F, Xu Z, Wang J, Chen Y, Li Q, Zuo Y, Chen J, Hu X, Zhou Q, Wang Y, et al: Recombinant human endostatin normalizes tumor vasculature and enhances radiation response in xenografted human nasopharyngeal carcinoma models. PLoS One 7: e34646, 2012.

22. Fukumura D and Jain RK: Tumor microvasculature and microenvironment: Targets for anti-angiogenesis and normalization. Microvasc Res 74: 72-84, 2007.

23. Kobayashi $\mathrm{H}$ and Lin PC: Antiangiogenic and radiotherapy for cancer treatment. Histol Histopathol 21: 1125-1134, 2006.
24. Han JY, Fan JY, Horie Y, Miura S, Cui DH, Ishii H, Hibi T, Tsuneki $\mathrm{H}$ and Kimura I: Ameliorating effects of compounds derived from Salvia miltiorrhiza root extract on microcirculatory disturbance and target organ injury by ischemia and reperfusion. Pharmacol Ther 117: 280-295, 2008.

25. Lei XL and Chiou GC: Cardiovascular pharmacology of Panax notoginseng (Burk) F.H. Chen and Salvia miltiorrhiza. Am J Chin Med 14: 145-152, 1986.

26. Wu L, Qiao H, Li Y and Li L: Protective roles of puerarin and Danshensu on acute ischemic myocardial injury in rats. Phytomedicine 14: 652-658, 2007.

27. Sugiyama A, Zhu BM, Takahara A, Satoh Y and Hashimoto K: Cardiac effects of salvia miltiorrhiza/dalbergia odorifera mixture, an intravenously applicable Chinese medicine widely used for patients with ischemic heart disease in China. Circ J 66: 182-184, 2002

28. Jin UH, Kang SK, Suh SJ, Hong SY, Park SD, Kim DW, Chang HW, Son JK, Lee SH, Son KH and Kim CH: Inhibitory effect of Salvia miltiorrhia BGE on matrix metalloproteinase-9 activity and migration of TNF-alpha-induced human aortic smooth muscle cells. Vascul Pharmacol 44: 345-353, 2006.

29. Tian XM, Wang R, Zhang BK, Wang CL, Guo H and Zhang SJ: Impact of Lycium Barbarum Polysaccharide and Danshensu on vascular endothelial growth factor in the process of retinal neovascularization of rabbit. Int J Ophthalmol 6: 59-61, 2013.

30. Cheng F, Wang X, Zhong D, Sun L, Wang Q and Liu C: Significance of detection of serum carbonic anhydrase IX in the diagnosis of lung cancer. Zhongguo Fei Ai Za Zhi 18: 29-33, 2015 (In Chinese).

31. Chen FH, Chiang CS, Wang CC, Tsai CS, Jung SM, Lee CC, McBride WH and Hong JH: Radiotherapy decreases vascular density and causes hypoxia with macrophage aggregation in TRAMP-C1 prostate tumors. Clin Cancer Res 15: 1721-1729, 2009.

32. Sundov Z, Tomic S, Alfirevic S, Sundov A, Capkun V, Nincevic Z, Nincevic J, Kunac N, Kontic M, Poljak N and Druzijanic N: Prognostic value of MVD, LVD and vascular invasion in lymph node-negative colon cancer. Hepatogastroenterology 60: 432-438, 2013.

33. Li J, Wang S, Yang X, Pang G, Zheng H, Shen B, Li G, Shi D, Wang J, Feng L, et al: Effect of sulfated polysaccharides from Laminaria japonica on vascular endothelial cells in psychological stress rats. J Ethnopharmacol 151: 601-608, 2014.

34. Wang ZJ, Yu YH, Chen J and Zhao YT: Correlation between oligohydramnios and abnormal expressions of TXA2, PGI2 and TXA2R in the umbilical arterial blood and placenta. Nan Fang Yi Ke Da Xue Xue Bao 29: 1917-1918, 2009 (In Chinese).

35. Zhang FH: Effects of acupuncture at 7-9 am and 3-5 pm on plasma thromboxane and prostaglandin in patients with ischemic cerebrovascular disease. J Tradit Chin Med 30: 9-12, 2010.

36. Liu HJ, Ma JW, Qiao ZY and Xu B: Study of molecular mechanism of Prostaglandin E1 in inhibiting coronary heart disease. Mol Biol Rep 40: 6701-6708, 2013. 